\title{
Distribución de las macroalgas en la laguna arrecifal de Banco Chinchorro: arrecife coralino del Caribe mexicano
}

\section{Distribution of macroalgae in the Banco Chinchorro reef Lagoon: coral reef of the Mexican Caribbean}

\author{
Alicia GonzÁlez ${ }^{1} \&$ Daniel Torruco² \\ Centro de Investigación y Estudios Avanzados del I.P.N. Unidad Mérida. Km 6 Antigua carretera a Progreso. A.P. \\ 73 CORDEMEX, Mérida, Yucatán, México. \\ 19aligonzalezsol@gmail.com, ${ }^{2}$ dantor6660@gmail.com
}

\begin{abstract}
RESUMEN
Se analizaron las macroalgas de fondos suaves de la laguna arrecifal de la Reserva de la Biosfera de Banco Chinchorro en junio de 2009, 2010 y 2011. El objetivo de la investigación fue conocer la estructura de las algas y sus cambios interanuales en una misma época. Se registraron 21 especies, Chondrophycus papillosus fue la dominante, los intervalos de diversidad estuvieron de 2,8 a 9,9 beles/ind. en los fondos arenosos; el área más diversa fue la parte media de la laguna. La similitud de localidades fue obtenida con el índice de Morisita y presentan un grupo heterogéneo que caracterizan áreas específicas dentro de la laguna arrecifal, principalmente cerca del borde de barlovento. El análisis canónico muestra la posición espacial de sitios y especies en relación a su dependencia de los factores del ambiente: la temperatura y la profundidad; la salinidad y el oxígeno disuelto no tienen influencia en las algas. Una muy alta correlación de 0,9 es presentada entre los parámetros del ambiente y los primeros dos componentes principales del sistema.
\end{abstract}

Palabras Clave: Macroalgas, arrecifes coralinos, distribución espacio-temporal, Caribe mexicano.

\begin{abstract}
The soft bottom macroalgae of the lagoon reef in the Biosphere Reservation of the Chinchorro Bank in june of 2009, 2010 and 2011 were analyzed. The research aim was to determine the algae structure and know their interannual changes in the same period. We registered 21 species Chondrophycus papillosus was the dominantest species, the intervals of diversity are from 2.8 to 9.9 beles/ ind, for sandy bottoms, the more diverse area was in the half part of the lagoon. The sites similarity was obtained with the Morisita's Index, data set present diverse and heterogeneous groups that characterized specific areas inside the reef lagoon, mainly the near ones to the windward reef. The canonical analysis shows the sampling sites and species space position and their environmental factors dependence: temperature and depth; the salinity and dissolved oxygen have not influence on algae. A high bigger correlation is presented at 0.9 between the environmental parameters and the first two main components of the system.
\end{abstract}

KEYWoRDS: Macroalgae, coral reef, spatial-temporal distribution, Mexican Caribbean.

\section{INTRODUCCIÓN}

Los arrecifes de coral son muy diversos y generalmente tienen una zonación muy definida. Los biotopos que se presentan en estas zonas son muy dinámicos y cambian constantemente con el tiempo, aun cuando sus límites espaciales pudieran son precisos (Bellwood et al. 2004). La presente investigación analiza la estructura de las algas arrecifales de Banco Chinchorro en el Estado de Quintana Roo, Caribe mexicano. La importancia de contribuir al conocimiento ficológico en áreas arrecifales, no solo es favorecer la elaboración de listas regionales sino que permite, a largo plazo, lograr un análisis biogeográfico y su subsecuente uso en el marco de la sistemática filogenética y la biología de la conservación. La naturaleza polifilética de este grupo implica una amplia diversidad que se manifiesta con 514 especies en el Caribe y el Golfo de México (Littler et al. 1989). Sin embargo; su conocimiento es escaso en áreas de arrecifes coralinos, donde existe un mosaico de ambientes con características diferentes de substratos, temperatura, radiación de luz y exposición al oleaje.

Por otro lado, un conocimiento detallado de descriptores ecológicos es de gran importancia para definir estrategias de conservación (Austin 1999) y utilización sostenida de áreas arrecifales, sobre todo porque permiten una identificación y valoración de las interacciones biológicas comunitarias más importantes con el ambiente, lo que lleva a una base de inicio robusta para la planeación y manejo integrado multisectorial de los recursos naturales en los arrecifes coralinos

Los antecedentes sobre el grupo en zonas cercanas al Banco o que corresponden a esta región caribeña de México, son variados tanto en objetivos, esfuerzo de muestreo, como 
en el tiempo (Huerta-Múzquiz 1958, Taylor 1972, GarzaBarrientos 1975, Jordan et al. 1978, Huerta-Múzquiz et al. 1987, Mateo-Cid \& Mendoza-González 1991, MendozaGonzález \& Mateo-Cid 1992, Aguilar-Rosas et al. 1992, Collado-Vides \& González-González 1993), sin embargo todos tienen un factor común, son listas de organismos que fueron colectados en las zonas medio y infralitoral del intermareal en variados momentos de tiempo.

Aun cuando los sistemas arrecifales son reconocidos por su enorme diversidad biológica y su importancia económica al ofrecer una gran variedad de bienes y servicios tanto ambientales como económicos (Torruco et al. 2013), están en un serio decremento globalmente, especialmente los que se encuentran cerca de plataformas continentales someras y densas poblaciones. Banco Chinchorro es un arrecife oceánico separado del continente; sin embargo es objeto de múltiples usos. Este arrecife en conjunto con los pertenecientes al Caribe, son la base de una industria turística que contribuye considerablemente en la economía de los países Caribeños; México para el 2012 fue considerado destino líder en la región al generar más del $30 \%$ del total de ingresos captando inversiones nacionales y extranjeras (Informe de Gobierno, 2012).

Específicamente en la laguna arrecifal del arrecife de Banco Chinchorro, la abundancia de las macrofitas en los alrededores de Cayo Centro (isla principal del complejo arrecifal) reviste gran importancia, si se considera el papel que juegan estos elementos como sitios de refugio, crianza, alimento y desarrollo de muchas especies que son elementales para el funcionamiento del arrecife (Torruco et al. 2003) y donde coexisten con otras que son de gran interés económicopesquero. De acuerdo con las consideraciones anteriores, el objetivo principal fue obtener un esquema de la distribución de las macroalgas durante el verano a lo largo de la laguna arrecifal del Banco, en una representación interanual.

\section{MATERIALES Y MÉTODOS}

Área de ESTUdio

Banco Chinchorro es un arrecife localizado al sureste de la Península de Yucatán, tiene la forma de atolón y está separado de la costa $24 \mathrm{~km}$ por un canal de $1000 \mathrm{~m}$ de profundidad (Fig. 1). Posee 4 islas: dos al norte conocidas como Cayo Norte, una central denominada Cayo Centro y la más pequeña en el sur, llamada Cayo Sur o Cayo Lobos. Posee gran variedad de hábitats que incluyen: una red de islotes de mangle en Cayo Centro y que ha originado un microsistema lagunar, una laguna arrecifal poco profunda con un gradiente batimétrico sur-norte de 13 a $2 \mathrm{~m}$ y parches arrecifales (microatolones) diseminados a lo largo de esta laguna (Torruco 1995). El arrecife tiene formaciones coralinas muy desarrolladas

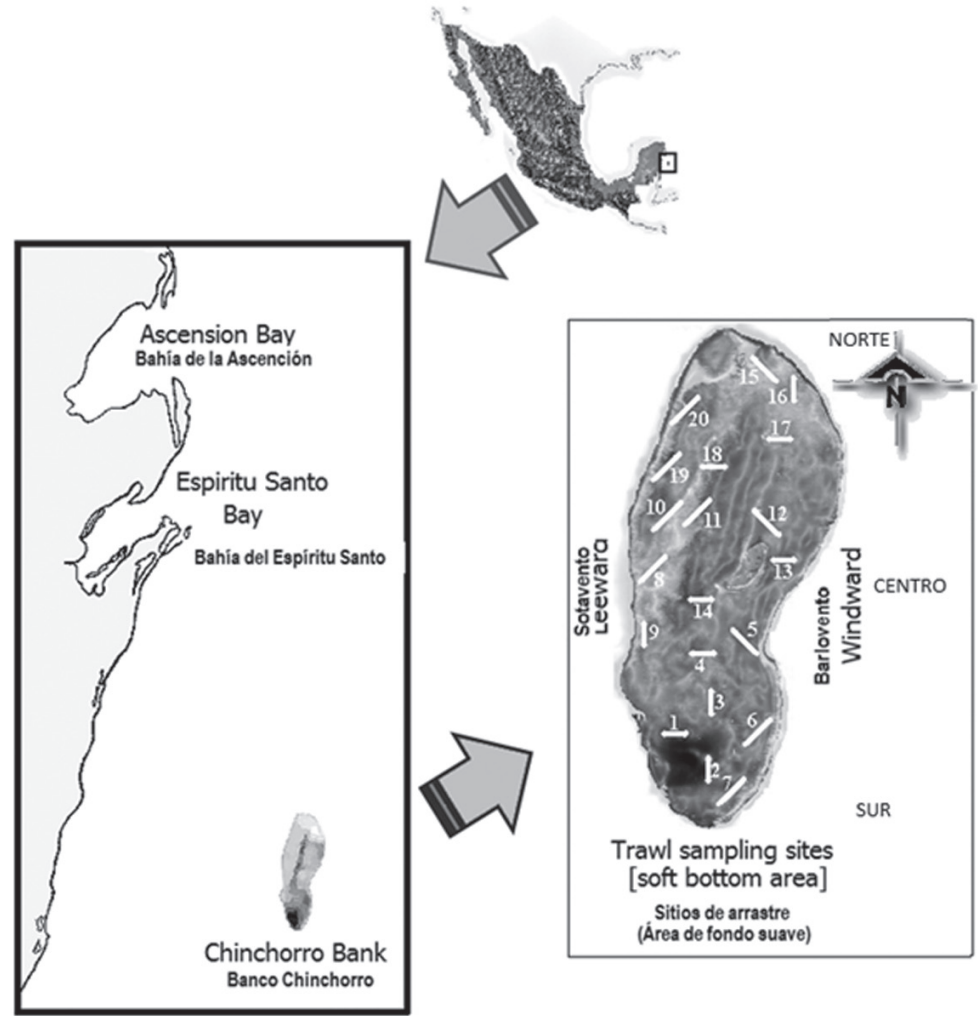

FiguRA 1. Localización del área de estudio. Se muestra la posición y orientación de los arrastres.

FigURE 1. Study area. It shows the position and orientation of trawling. 
en la parte sur y en barlovento y pendientes muy abruptas en sotavento que exceden los $500 \mathrm{~m}$. Es el más grande de México, el área total de la laguna arrecifal y la rompiente es de $814,2 \mathrm{~km}^{2}$ (Torruco et al. 2003).

\section{RECOLECTA}

La recolecta de macroalgas se realizó en la laguna arrecifal en 20 sitios específicos (fondos no coralinos susceptibles de usar draga) mediante arrastres de $15 \mathrm{~min}$ de duración con una pequeña red de arrastre de $1000 \mathrm{~cm}^{2}(50 \times 20 \mathrm{~cm})$ de área en la boca del colector y con una velocidad de 0.5 nudos, durante los años 2009, 2010 y 2011, cubriendo un intervalo de profundidad de 2 a 10 m (Fig. 1). Todos los muestreos fueron realizados en el verano en el mes de junio. Las muestras fueron conservadas en formalina para su transporte al laboratorio y posterior a la identificación se obtuvo el porcentaje de biomasa seca de cada especie, a excepción de las epifitas, las cuales se contaron como un solo grupo (Tabla I). Antes de cada arrastre se registraron: temperatura, salinidad y oxígeno disuelto tanto superficial como a media agua $(5 \mathrm{~m})$, utilizando una sonda multiparámetro YSI-185. La profundidad de cada sitio fue obtenido con un profundímetro digital Hondex PS-7.

Tabla I. Resultados de la Prueba de Bartlett y Cochran.

TABLE I. Test Bartlett and Cochran.

\begin{tabular}{lcccc} 
Hartley & Cochran & Bartlett & GL & P \\
\hline F-max & C & Chi-Cua & & \\
1,510535 & 0,394226 & 0,370836 & 3 & 0,830757
\end{tabular}

ANÁLISIS

Al momento de ser identificados se registró la forma de los ejemplares colectados para conocer la variedad morfológica de esta flora. Su análisis se efectuó bajo dos criterios: un análisis global con las 20 estaciones distribuidas en todo el Banco y otro dividiendo la laguna arrecifal en tres áreas: norte, centro y sur. Con el primero se busca detectar las similitudes entre todas las estaciones y en el segundo establecer si existen diferencias significativas en cada área dada la extensión de la zona lagunar. Con ello, se pretende representar un esquema interanual detallado de la configuración espacial de la flora sumergida en la laguna arrecifal de Banco Chinchorro, ya que la dinámica del arrecife y la mayor utilización se presenta en esta área.

El análisis numérico realizado incluye: Inicialmente un análisis de varianza (ANDEVA) de una vía (Marques 1998) para comparar si existen diferencias significativas entre cada año tanto para la riqueza de especies como para la biomasa; posteriormente, se obtuvo la dominancia bajo el criterio jerárquico de Orloci (1978); la diversidad ecológica con el índice de Simpson (Magurran 1988). El ensamblaje de las estaciones fue obtenido con el índice de similitud de Morisita con el criterio de unión flexible con una $\beta=0.25$ (Pielou 1984).

Finalmente se utilizó una ordenación n-dimensional de sitios mediante un análisis de Componentes Principales (ACP) y una ordenación de sitios y especies en relación a los factores del ambiente mediante un análisis de Correlación Canónica (ACC) usando el programa STATISTICA 9.0 (StatSoft 2010), con la intención de conocer si alguno de estos factores influía de manera determinante en la posición espacial de los sitios y de las especies y si podría presentarse algún patrón en ellos.

\section{RESULTADOS}

Se verificaron los supuestos de homocedasticidad y normalidad mediante las pruebas de Bartlett y Cochran y Kolmogorov y Smirnov respectivamente (Zar 1999), p>0,05, se asumió que las varianzas son homogéneas y se verificó que la distribución normal y la que exhiben los dos descriptores no muestran diferencias significativas (Tabla I). Al realizar el ANDEVA ( $>0,05)$ se aceptó que en los 3 años de muestreo no existieron diferencias significativas ni en el número de especies ni en la biomasa (Tabla II), ya que ambas manifestaron fluctuaciones mínimas (de 2 a 3 especies y de 5 a 8 gr); sin embargo, hubo una cierta sustitución en la identidad de las especies más frecuentes en cada uno de los años. Con este resultado, los datos fueron estandarizados y tratados como una sola matriz.

TABLA II. ANDEVA de una vía para la comparación de los tres años de muestreo.

TABLE II. Way ANOVA for comparison of the three years of sampling.

\begin{tabular}{lcrccc} 
EғECTо & Ss & GL & Ms & F & P \\
\hline Año/riqueza & 0,001146 & 3 & 0,000573 & 0,7812 & 0,467578 \\
Error & 0,017302 & 28 & 0,000618 & & \\
Año/Biomasa & 0,000067 & 3 & 0,000033 & 1,0589 & 0,368643 \\
Error & 0,000535 & 17 & 0,000031 & &
\end{tabular}

La biomasa total y la dominancia total y por zonas de la flora arrecifal están desplegadas en la Tabla III. Chondrophycus papillosus fue la macroalga con mayor dominancia en las zonas someras de todo el Banco; seguida de Lobophora variegata, Udotea flabellum y Halimeda incrassata. Las demás especies tienen valores de dominancia pequeños. Sin embargo, la dominancia que presenta Chondrophycus papillosus sólo se refleja en la zona Central ya que en las otras dos zonas la jerarquía de esta especie se difunde y son otras las que presentan los valores más altos. Algunas especies como Amphiroa fragilissima y Caulerpa cupressoides, a pesar de presentarse en las tres zonas, su dominancia es muy baja.

La diversidad más alta corresponde a los sitios 12 y 6 con 9,9 y 7,9 beles/ind. respectivamente y la más baja a los sitios 1 y 3 (2-8 beles/ind.) (Tabla IV). La profundidad en general nunca rebasó los $10 \mathrm{~m}$. La equidad más alta se localizó en la estación 19 y la más baja en la estación 11 . 
TABLA III. Morfología, biomasa y dominancia de especies dada por el índice de Orloci para las macroalgas de Banco Chinchorro, arrecife del Caribe mexicano.

TABLE III. Morphology, biomass and dominance (Orloci index) of macroalgae in Chinchorro Bank, Mexican Caribbean reef.

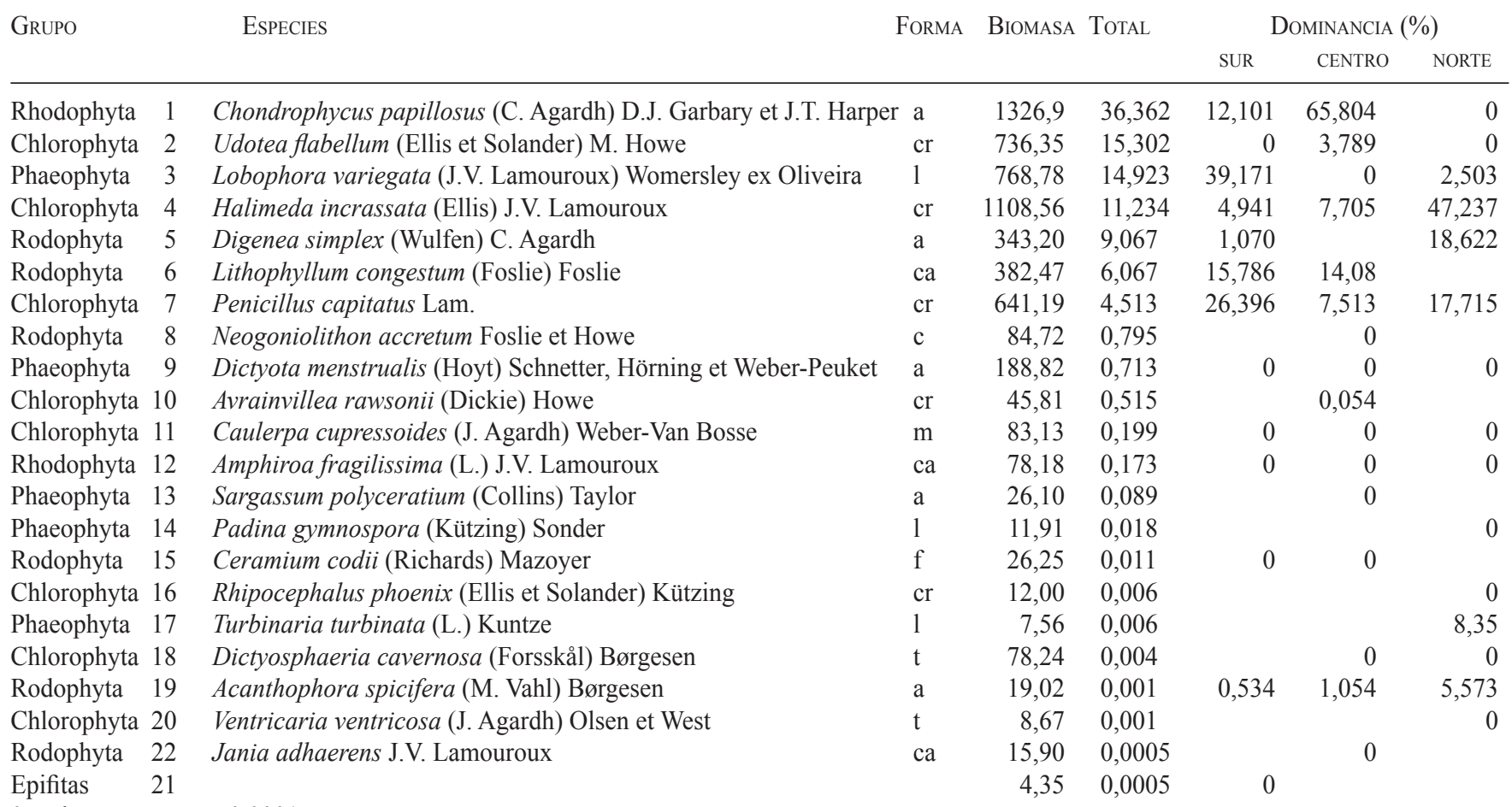

0 : valores menores a 0,0001

a: arbustivas, ca: calcáreas articuladas, cr: calcárea rizoidales, c: costrosas, f: filamentosas, 1: hojas, m: modulares, t: tapete

Si se homologa el corte del análisis de similitud con la regla para identificar provincias zoogeográficas que menciona que por lo menos el 50\% de especies deben ser nativas, los sitios que contengan menos del $50 \%$ de similitud deben considerarse diferentes, en este caso las macroalgas conforman 5 grupos a ese nivel de corte. El grupo I incluye el sitio 1 y 18 muy cercanos a los extremos sur y norte; el grupo II caracteriza la zona de barlovento, sus sitios están emplazados en esa orilla; el grupo III es el más grande y está formado por estaciones diseminadas principalmente en la zona Central y una del Norte del Banco; el grupo IV es muy heterogéneo, presenta cuatro sitios: dos de la parte sur y dos de la parte norte; el último grupo (V) asocia dos sitios: el más sureño y uno de la parte media estaciones, pero ambos en la línea de barlovento (Fig. 2a).

El análisis de componentes principales discrimina un poco más los grupos obtenidos con la clasificación numérica, aunque también se definen 5 grupos (Fig. 2b) y 4 sitios solitarios. El primero caracteriza una zona central al sur de Cayo Sur. El segundo reúne sitios muy cercanos a los bordes de la laguna en ambos lados (barlovento y sotavento). El tercero muestra estaciones centrales un poco cargadas hacia barlovento, la afinidad de la estación 17 la acerca a este grupo sin pertenecer propiamente a él. El cuarto grupo reúne elementos del centro de la laguna arrecifal, con excepción de la estación 20 que se ubica hacia la parte noreste del Banco. El último grupo reúne la estación 1 y la 18.
TABLa IV. Estimaciones de la riqueza específica (S), diversidad (D), equidad (E) y profundidad (P) de las 20 estaciones de arrastre en Banco Chinchorro, Arrecife del Caribe Mexicano. Se presentan las diversidades máximas (Dmáx) y mínimas (Dmín).

TABLE IV. Species richness (S), diversity (D), evenness (E) and depth (P) in the 20 trawl sites in Chinchorro Bank, Mexican Caribbean reef. It shows the maximal and minimal diversities.

\begin{tabular}{lrrrrrl} 
Sitios & S & D & DмÁx & Dмі́N & E & P (м) \\
\hline 1 & 5 & 2,8 & 5,211 & 1,086 & 0,370 & 5 \\
2 & 7 & 4,3 & 7,452 & 1,132 & 0,396 & 6 \\
3 & 3 & 2,8 & 3,062 & 1,042 & 0,631 & 6 \\
4 & 8 & 6,2 & 8,609 & 1,157 & 0,498 & 6 \\
5 & 5 & 4,9 & 5,211 & 1,086 & 0,657 & 6 \\
6 & 11 & 7,9 & 12,237 & 1,236 & 0,448 & 2,5 \\
7 & 5 & 4,2 & 5,211 & 1,086 & 0,563 & 5 \\
8 & 8 & 4,4 & 8,609 & 1,157 & 0,354 & 7 \\
9 & 6 & 3,3 & 6,319 & 1,109 & 0,363 & 4,5 \\
10 & 6 & 5,5 & 6,319 & 1,109 & 0,609 & 3,5 \\
11 & 8 & 2,9 & 8,609 & 1,157 & 0,237 & 4,5 \\
12 & 10 & 9,9 & 11,008 & 1,211 & 0,629 & 6,5 \\
13 & 8 & 4,5 & 8,609 & 1,157 & 0,362 & 4 \\
14 & 8 & 6,5 & 8,609 & 1,157 & 0,526 & 5 \\
15 & 7 & 4,9 & 7,452 & 1,132 & 0,460 & 2 \\
16 & 6 & 5,8 & 6,319 & 1,109 & 0,630 & 4 \\
17 & 7 & 3,8 & 7,452 & 1,132 & 0,358 & 4 \\
18 & 5 & 4,1 & 5,211 & 1,086 & 0,544 & 6 \\
19 & 4 & 4,9 & 4,125 & 1,063 & 0,819 & 5 \\
20 & 6 & 3,5 & 6,336 & 1,114 & 0,360 & 5
\end{tabular}



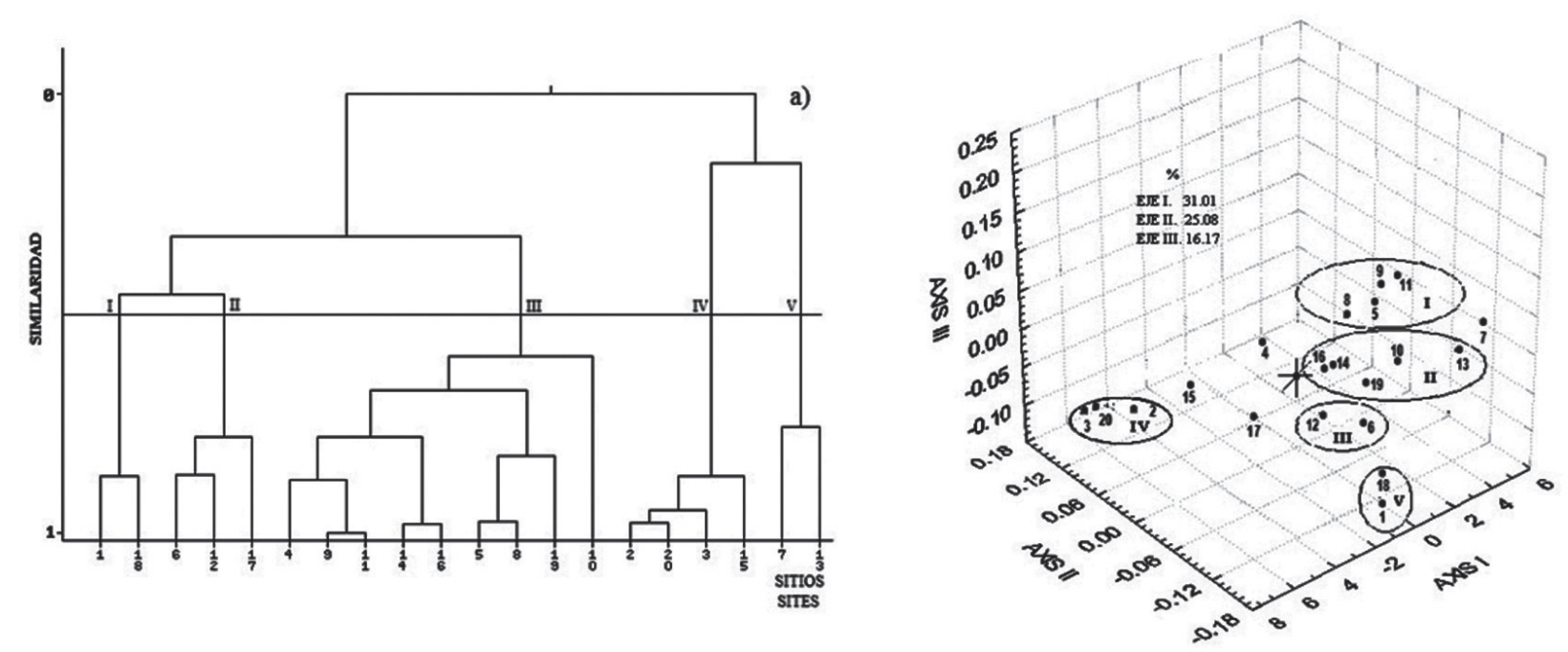

b)

Figura 2. Similitud y posición espacial de sitios en la laguna arrecifal de Banco Chinchorro. a) El dendrograma de similitud global, bajo el criterio del índice para proporciones de Morisita en las estaciones de muestreo, muestra 5 grupos: el I aglutina las estaciones más profundas; el II caracteriza sitios de barlovento; el III recluta sitios de la zona media; el IV muestra sitios en ambos extremos y el V también reúne sitios de la orilla de barlovento. b) La Ordenación tridimensional de los sitios por el ACP redefine los grupos anteriores y presenta 5 grupos: El I caracteriza el lado de sotavento; El II muestra sitios tanto de barlovento como de sotavento restringidos a la parte central y norte; el III une sitios de barlovento; el IV y el V congregan sitios de ambos extremos. Se presenta el porcentaje $(72,26)$ de la varianza explicada en los tres ejes de mayor importancia.

FIgURE 2. Similarity and spatial position of sites on the Chinchorro Bank lagoon. a) The Morisita index similarity dendrogram in all sampling sites, shows 5 groups: the I brings together the deepest stations; II characterized Windward sites; the III recruits central sites; the IV sample sites at both ends; and V also meets windward shore sites. b) The ordination three-dimensional of sites by the ACP presents five groups: the I, characterized the leeward side, the II shows both sides windward and leeward restricted to the central and northern part, the III joins windward sites, the IV and V congregate sites of both ends. Show the percentage $(72,26)$ of the explained variance in the three axes of greatest importance.
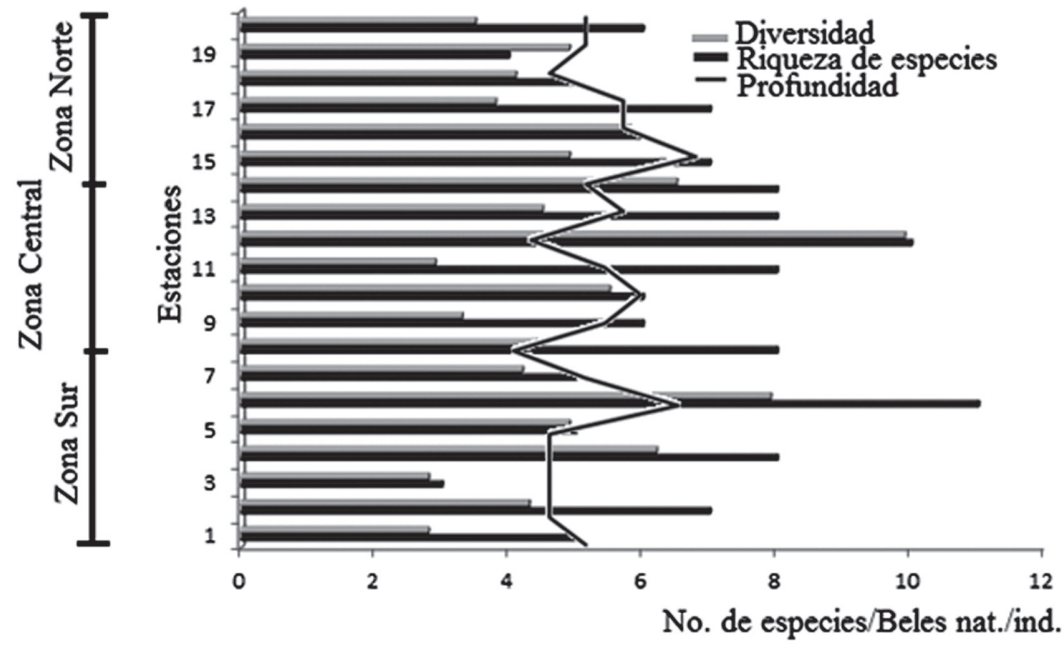

Figura 3. Diversidad (índice de Simpson) de las algas en la laguna de Banco Chinchorro y la profundidad de los sitios de muestreo. Zona Sur. Se muestra un pico de diversidad en el sitio 6, que corresponde al más somero. Zona Central. Se presenta el mayor pico de diversidad en el sitio 12 muy cercano a la isla Cayo Centro. Zona Norte. Se muestra estaciones con diversidades homogéneas.

Figure 3. Simpson index diversity of algae in the Chinchorro Bank lagoon and depth of sampling sites. South Zone. Shows a diversity peak at the site 6, which corresponds to the shallowest. Central Zone. It has the highest peak of diversity at site 12 very close to the Cayo Centro island. North Zone. Diversities shown homogeneous stations. 

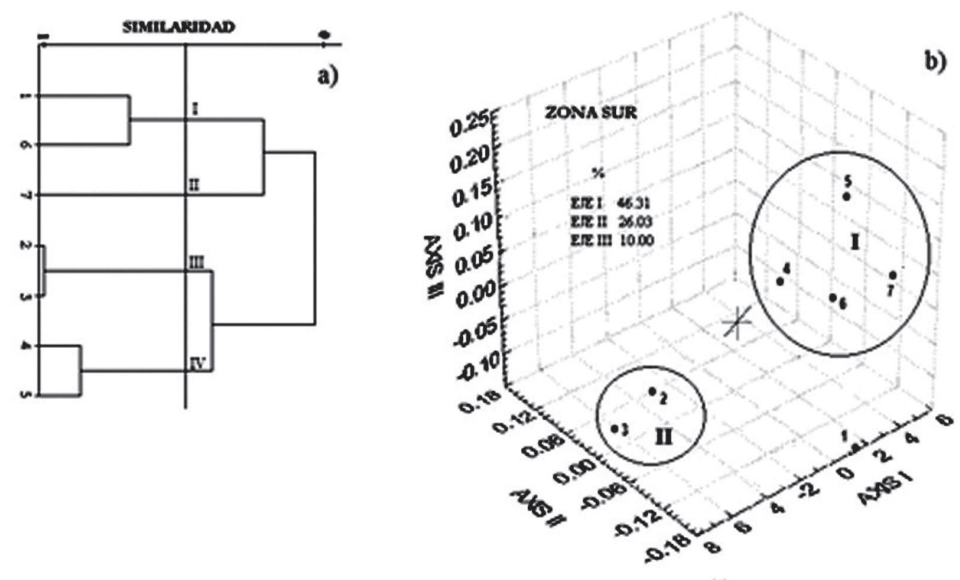

b)
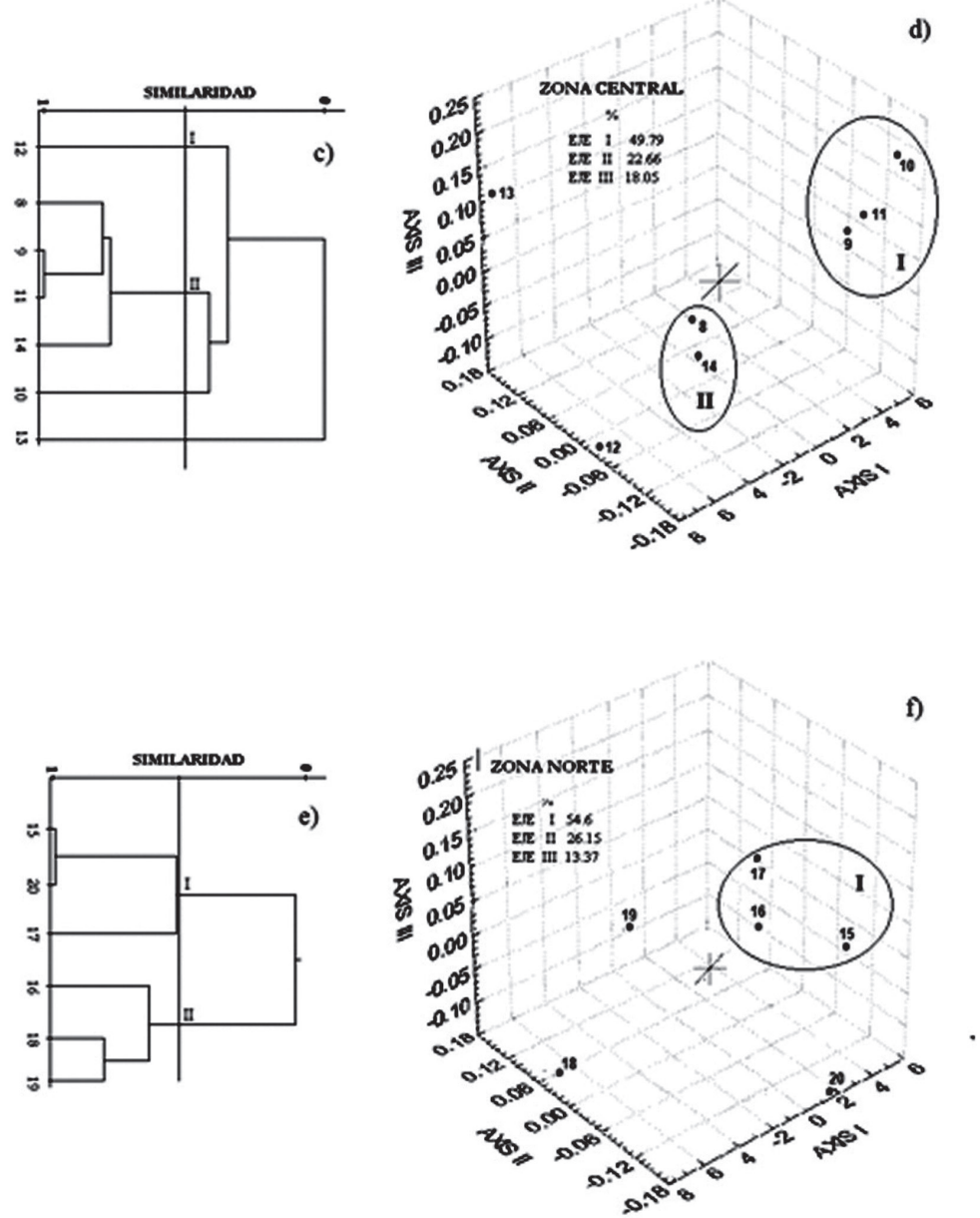

Figura 4. Similitud y posición espacial de sitios por zonas en la laguna arrecifal de Banco Chinchorro. a) Zona sur: la similitud crea 3 grupos que se unen principalmente en función de la profundidad. b) El ACP muestra 2 grupos: el primero define el lado de sotavento y el segundo la parte media. c) Zona Central: la similitud forma un solo grupo que caracteriza el lado de sotavento y el centro de la laguna, los otros sitios quedan solitarios. d) El ACP constituye dos grupos el primero caracteriza el lado de sotavento y el segundo el centro de la laguna. e) Zona Norte: forma dos grupos que caracterizan la punta norte y una franja que cruza la laguna. f) el ACP integra un solo grupo que caracteriza el lado de barlovento.

Figure 4. Similarity and spatial location of sites for areas in Chinchorro Bank lagoon. a) South Zone: the similarity creates 3 groups that bind primarily as a function of depth. b) The ACP shows 2 groups: the first defines the leeward side and the second the middle of the lagoon. c) Central Zone: the similarity form a single group that characterizes the leeward side and the center of the lagoon, the other sites are loners. d) The ACP outline two groups, the first one characterized the leeward side and the second the center of the lagoon. e) North: develop two groups form the northern tip and a strip that crosses the lagoon. f). The ACP integrates a single group that characterizes the windward side. 
El esquema que se presenta por zonas es el siguiente: Zona SUR: Presenta una dominancia de Lobophora variegata, seguida de Penicillus capitatus, Litophyllum congestum, y Chondrophycus papillosus. Las especies restantes tienen dominancias mínimas (Tabla III). La diversidad de este grupo es más alta en las estaciones 6 y 4 con 7,91 y 6,18 bits/ind respectivamente (Fig. 2a).

El análisis de agrupamiento y siguiendo el mismo criterio de corte para ésta zona forma tres grupos que caracterizan tanto al borde de la laguna arrecifal como a la zona Central; esta última a su vez se subdivide en 2 áreas, una con tendencia sureña y otra que va hacia el Centro (Fig. 4a). El ACP define dos grupos, el primero determina la zona de barlovento en esa área y la segunda una zona central; el sitio, 1 queda como una entidad solitaria (Fig. 4b). La varianza que explican los tres ejes principales es de $82,34 \%$ del total.

Zona CENTRAL: Chondrophycus papillosus es el elemento dominante; por lo que, influye en el análisis total, todas las demás especies tienen valores más bajos (Tabla III). La diversidad presenta un pico de gran magnitud en la estación 12 (localizada al norte del Cayo), siendo también una de las áreas más profundas de esta zona y la de mayor equidad (Fig. 2b). El dendrograma muestra la formación de un solo grupo de sitios que comprende áreas ubicadas en sotavento en esa área; las otras estaciones se unen de manera individual a niveles de similitud más bajos (Fig. 4c). En la ordenación multifactorial se obtienen 2 agrupaciones, la primera une las estaciones 9, 10 y 11 que caracterizan el borde de sotavento en la porción Central del Banco, mientras que el segundo une áreas en barlovento. Las estaciones 12 y 13 mantienen su identidad ya que no se acercan a ningún conglomerado (Fig. 4d). La varianza que explican los tres ejes principales es de 90,5\%.

ZONA NORTE: Halimeda incrassata es la especie dominante (reafirmando su papel como formadora y consolidadora de substrato), seguida de Lobophora variegata, las otras especies tienen valores menores (Tabla III). La diversidad más alta para esta área la presenta la estación 16 ubicada muy cerca a la rompiente arrecifal de barlovento; mientras que la más baja se localiza cercana a la rompiente de sotavento (Fig. 2c). El análisis de similitud muestra dos grupos bien diferenciados de 3 estaciones cada uno. Esta asociación incluye las estaciones 15, 16 y 17 que caracterizan la punta Norte del banco. Las estaciones restantes $(16,18$ y 19$)$ tipifican la parte central de la zona norte; no obstante sus propias características intrínsecas no les permite una mayor similitud y se unen a valores bajos (Fig. 4e). El ACP identifica un solo grupo que caracteriza la zona de barlovento $(15,16$ y 17) y presenta a los otros 3 sitios separados totalmente (Fig. 4f). La varianza que explican los tres ejes principales es de $94,12 \%$.

El análisis de Correlación Canónica muestra la influencia de la temperatura y de la profundidad sobre sitios y especies y la poca influencia que la salinidad y el oxígeno tienen en ese patrón espacial (Fig. 5). Tanto los sitios como las especies se ordenan en un gradiente que direcciona la profundidad y la temperatura tanto superficial como a $5 \mathrm{~m}$. La salinidad y el oxígeno no influyen en ese gradiente.

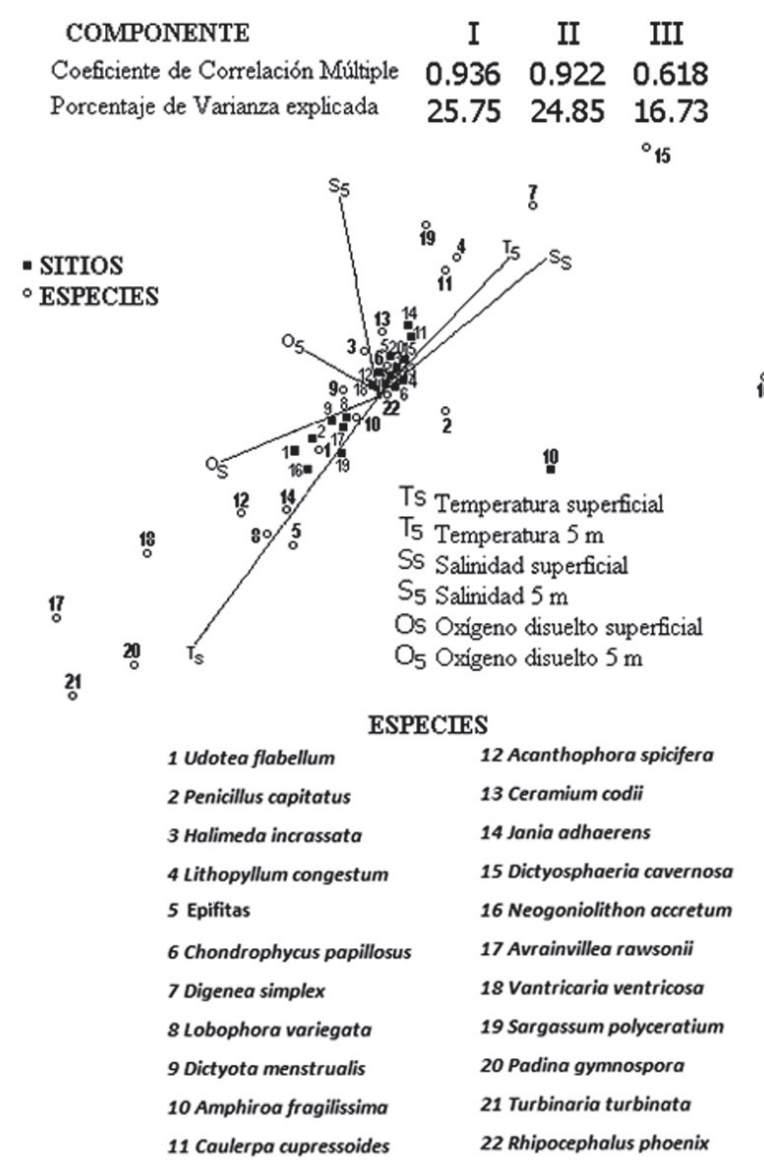

Figura 5. El análisis de Correlación Canónica muestra un gradiente tanto de sitios como de especies a lo largo de los ejes de temperatura como de profundidad. Los coeficientes de correlación son muy altos en los dos primero ejes $(>0,9)$ y la varianza explicada por los tres primeros es de $67,71 \%$.

Figure 5. Canonical Correlation analysis shows a gradient of both sites and species along the axes of temperature and depth. The correlation coefficients are very high in the first two axes $(>0.9)$ and the variance explained by the first three is $67.71 \%$.

\section{DISCUSIÓN}

La falta de diferencias significativas en los volúmenes de biomasa de los tres años de muestreo fue reflejo de que, en esos años para el mes de junio, no hubo grandes cambios ambientales; los registros de temperatura, vientos y otros elementos del clima no presentaron diferencias significativas, incluso desde el mes anterior; frecuentemente en esa época, se presenta la temporada de huracanes y tormentas tropicales, que consecuentemente podrían afectar la estructura de 
las algas. Desafortunadamente en estos tres años no hubo huracanes o tormentas considerables en ésta época para ver cambios conspicuos en la estructura algal. Esto no significa que no se hayan presentado cambios a lo largo de los años, sino que en un análisis interanual en la misma época no existieron cambios estadísticamente significativos; sin embargo, sí se presentaron cambios ecológicos, ya que hubo cambios sutiles en la representación de algunas especies y su reemplazo por otras, tanto en la dominancia como en la cantidad de biomasa.

La frecuencia de la morfología de las especies registradas identifica las características heterogéneas del substrato y su alternancia en los diferentes sitios de muestreo. La riqueza de especies comparada con otras Áreas Naturales Protegidas (ANP's) arrecifales es baja (Tabla V), incluso la reportada para este mismo arrecife (INE 2000); sin embargo, se deben tener en cuenta varios argumentos: las listas que se presentan en los otros trabajos son compilaciones variadas, realizadas en diferentes épocas del año y bajo diferentes esquemas y metodologías de trabajo; en nuestro caso, solo nos referimos a las macroalgas susceptibles de ser colectadas mediante arrastres y solo en la laguna arrecifal, no se realizaron muestreos en la zonas coralinas ni en las zonas de surcos y crestas del arrecife posterior, que también poseen una gran diversidad de flora, donde la estabilidad de los substratos permite el desarrollo de un número mayor de formas algales como lo han comprobado Taylor (1972) y Mateo-Cid \& Mendoza-González (1991).

Para el área de fondos suaves, los resultados muestran que la flora marina de Banco Chinchorro es diversa, alcanzando valores de 9,9 beles/ind, en la parte central, cercana a Cayo Centro. Esta diversidad podría deberse a que en la mayoría de las zonas muestreadas existe una combinación de facie rocosa manifestada como fragmentos coralinos y arena gruesa, lo que proporciona una gran variedad de hábitats, donde pueden desarrollarse diferentes especies de macroalgas; las morfologías más representadas son arbustivas y calcáreas rizoidales, que necesariamente necesitan un substrato duro en el caso de las primeras y un sustrato arenoso por parte de las segundas; las menos frecuentes son las costrosas y las modulares, en el primer caso porque no existe un sustrato sólido lo suficientemente amplio, como en la rompiente arrecifal, donde pueda desarrollarse este tipo de algas. El factor que parece tener mayor influencia en la diversidad de las macroalgas de fondos suaves es la profundidad, donde áreas más someras presentan una mayor diversidad (Hughes 1994).

El grupo de las epifitas fue realmente pequeño en comparación a lo encontrado en las costas del Estado de Quintana Roo por Garza-Barrientos (1975), Huerta-Múzquiz (1986), Aguilar-Rosas et al. (1998), Mateo-Cid \& MendozaGonzález (1991), quienes manifiestan que es una situación común en la flora regional; la diferencia podría estar relacionada en la característica de los substratos, ya que las investigaciones citadas anteriormente se refieren a substratos duros, a una escasa profundidad $(<2 \mathrm{~m})$ y a zonas con cierta protección al oleaje, mientras que nuestro trabajo se realizó en zonas de substrato suave.

De acuerdo al análisis de afinidad, la zona más identificable ha sido barlovento y si algunos sitios del centro o de la parte de sotavento se unen con ésta, puede deberse a las características de relleno (azolve) que se presentan en esta zona, lo que la asemeja a las llanuras arenosas de la parte central. Otras, sin embargo, se unen posiblemente por la etapa de desarrollo de la vegetación sumergida en esas áreas (Hughes et al. 1999).

La discrepancia en los sitios de sotavento de la zona Norte hace que se separen drásticamente, esto es un indicio de la heterogeneidad que presenta esta zona, sobre todo en lo relacionado a las características del substrato y la profundidad; sin embargo, es probable que la mayor diversidad registrada en barlovento sea originada a las condiciones de oleaje más inalterable y dúctil, así como al mayor número de zonas con cierta protección a esta variable. Los factores que controlan la abundancia de macroalgas en el Caribe han sido discutidos por varios autores (Chapman 1961, Huerta 1986, Littler et al. 1987, Lapointe 1997); estudios recientes y observaciones en otros arrecifes sugieren que los arrecifes no son estables en sus asociaciones interespecíficas en un supuesto equilibrio como se postulaba anteriormente (Connell 1976, Littler et al. 1993), esta observación es ampliamente corroborada con las macroalgas de Banco Chinchorro.

Es conveniente resaltar que las condiciones ciclónicas frecuentes en esta zona (Lugo et al. 2000, Islebe et al. 2009), originan disturbios ecológicos de gran magnitud para las

TABLA V. Comparación de la riqueza de especies de algas en otras Áreas Naturales Protegidas en la región del Caribe mexicano. TABLE V. Algae richness comparison with other National Protected Area in the Mexican Caribbean Region.

\begin{tabular}{lccrrr} 
Área natural Protegida & Chlorophyta & Phaeophyta & Rodophyta & Totales & Fuente \\
\hline Sian Ka'an & 66 & 19 & 86 & 171 & INE, 1996 \\
Isla Contoy & - & - & - & 41 & INE, 1997 \\
Costa Occidental de I. Mujeres, P. Cancún y P. Nizuc & 86 & 44 & 124 & 264 & INE, $1998 \mathrm{a}$ \\
Cozumel & 75 & 55 & 166 & 296 & INE, $1998 \mathrm{~b}$ \\
Sian Ka'an & 15 & 46 & 35 & 96 & Keeney, 1999 \\
Banco Chinchorro & 59 & 22 & 53 & 135 & INE, 2000 \\
Banco Chinchorro & 19 & 11 & 18 & 38 & This work
\end{tabular}


macroalgas de estas zonas, sobre todo para las localizadas en la parte de barlovento del atolón. Tales disturbios causan grandes cambios en sus poblaciones debido a efectos directos como la intensidad del oleaje (Tunnichffe 1983), e indirectos como el aumento de sedimentos en suspensión que eventualmente se depositan sobre la flora (Boem 1988); y aunque no hubo diferencias significativas a lo largo de los tres años de muestreo en el mes de colecta, la alta correspondencia de los sitios en la orilla de barlovento pueden ser efecto de esta influencia.

Por otro lado, si bien el arrecife es un sistema saludable de acuerdo a los estándares establecidos por la línea base del Sistema Arrecifal Mesoamericano (SAM) (García et al. 2006), los impactos originados por la utilización de sus cayos arenosos como base pesquera de las cooperativas que se dedican a la pesca del caracol rosado y la langosta, aunado el uso turístico con escasa legislación reglamentaria podrían ser los responsables de los principales perjuicios (disminución de la diversidad, decremento en la cobertura de elementos sensibles del arrecife, eutrofización por desechos de fileteado de peces, etc.) que pudiera ocasionarse en este sistema, sobre todo que la laguna arrecifal es la zona de mayor uso de ambas industrias (Balám-Dzul \& De Jesús-Navarrete 2011).

A pesar de esto y debido a las pequeñas magnitudes de impacto ecológico registrados, la mayoría de los patrones de distribución de macroalgas deben ser atribuidos a los efectos de la variabilidad del ambiente, una diferencia obvia entre las diversas zonas de Banco Chinchorro es el gradiente de profundidad presentado, no así la temperatura. Estas tendencias en la actualidad pueden estar relacionadas a factores como la disponibilidad de luz, alta sedimentación y especificidad del substrato, ya que estos elementos podrían ser los que presentan mayor importancia en estas comunidades (Bradbury \& Young 1981, Littler \& Littler 1985), desafortunadamente el presente análisis no involucra un examen detallado de estos factores; sin embargo, se realizó un análisis indirecto (ACC), al comparar la batimetría, la temperatura, la salinidad y el oxígeno disuelto como los causantes directos de estos patrones, lo que dio como resultado la revelación de la importancia que juega el efecto concatenado de la profundidad y la temperatura en el patrón espacial de los sitios y de las especies. El alejamiento de la especie Turbinaria turbinata que muestra este análisis se debe principalmente a que prefiere sitios expuestos al oleaje su desarrollo en substratos duros que en este caso son fragmentos de coral muerto. Así, se percibe que los substratos definen agrupaciones de sitios y especies, mientras que las condiciones oceanográficas lo permitan y éstas a su vez definen agrupaciones de deriva algal.

Algunas especies como Halimeda se caracterizan por presentar un sistema rizoidal y una morfología compacta, lo que le confiere cierta ventaja para resistir oleajes intensos (Mata 1981), otras sobreviven a la abrasión del sedimento en áreas con gran energía, debido a su capacidad para colonizar hábitats crípticos. La dominancia de Halimeda incrassata en la zona Norte, es evidencia real de estas situaciones. Además, su comportamiento como ocupadora de espacio y trampa para el sedimento no consolidado de esta especie también ha sido ampliamente documentado en arrecifes de Jamaica (Liddell et al. 1988).

El análisis de clasificación conduce a reconocer las diferencias cuantitativas entre las diferentes zonas, extrae las consideraciones subjetivas y descubre la importancia de los atributos ecológicos identificados en el campo. Sin embargo, en algunos casos los factores que originan los patrones de distribución no son únicos y no se descubren con claridad, aun cuando los análisis complementarios realizados sugieren que ocurren cambios significativos con la profundidad y la temperatura, muestra además que las diferentes partes de un mismo sistema arrecifal pueden estar sujetas a distintas presiones y combinaciones en el proceso de selección, aun en áreas fisiográficamente relacionadas con la frecuencia e intensidad de disturbio por el oleaje como son las zonas de barlovento y sotavento (Ezer et al. 2005). Esto implica que la biodiversidad de las algas presenta un comportamiento dinámico relacionado con las condiciones del lugar y a los ciclos estacionales de estas condiciones.

Un aspecto que se debe tener en cuenta es la naturaleza de parche (espacial) de muchas especies de algas, que podría observarse como un aumento progresivo en la cobertura algal hacia las islas. Esto último puede aparecer como un resultado originado por las fluctuaciones en las tasas de crecimiento debidas a la variación en la disponibilidad de nutrientes, sobre todo si se considera la naturaleza cárstica de la Península de Yucatán y la intrusión de aguas ricas en nutrientes en la laguna arrecifal de Banco Chinchorro que provienen del microsistema lagunar que se presenta en Cayo Centro, la isla más grande del arrecife. Lo anterior induce la formación de surgencias locales que tienen una influencia marcada en la nutriclina de profundidades someras y proporcionan ambientes propicios para el crecimiento de la flora béntica, y que ocasionan un aprovechamiento intenso por las algas y una rápida proliferación de ellas (Crossland et al. 1984). Este aporte de nutrimentos no solo influye en el rápido crecimiento, sino también en la forma de crecimiento, en la propia morfología de las especies y en el estatus reproductivo (Wynne 2002).

Una particularidad interesante es la distribución de peces herbívoros en esa área, que por ser muy somera son escasos y donde no se registró ningún impacto provocado por esa fauna, aun cuando algunas investigaciones mencionan algunas especies herbívoras como abundantes en este sistema arrecifal (Núñez-Lara \& Arias-González 1998). El estudio de la distribución de este grupo y de invertebrados herbívoros podría explicar los sutiles cambios de dominancia entre las especies de algas en intervalos interanuales, donde los parámetros del ambiente se mantienen sin cambios significativos. 


\section{BIBLIOGRAFÍA}

Aguilar-Rosas, L.E., M.A. Aguilar-Rosas, A. Gómez-PedrosoCedillo \& J.A. Fernández-Prieto. 1992. Adiciones a la flora marina del Caribe mexicano. Acta Botánica Mexicana 19: 77-84.

Aguilar-Rosas, M.A., L.C. Aguilar-Rosas \& R. Aguilar-Rosas. 1998. Algas marinas de la región central de Quintana Roo. Paleobotánica 7: 15-32. IPN.

Austin M.P. 1999. The potential contribution of vegetation ecology to biodiversity research. Ecography 22: 465-484.

Balám-Dzul, V. \& A. De Jesús-Navarrete. 2011. Densidad, abundancia y estructura poblacional del caracol blanco Strombus costatus en el Caribe Mexicano. Revista de Biología Marina y Oceanografía. 46(1): 1-8.

Bellwood, D.R, T.P. Hughes, C. Folke \& M. Nyström. 2004. Confronting the coral reef crisis. Nature 429: 827-833.

Boem, R.M. 1988. Recognition of strom impact on the reef sediment record. Proceeding of the 6th International Coral Reef Symposium, Australia. 2: 475-478.

Bradbury, R. H. \& P. C. Young. 1981. The effects of a major forcing function, wave energy, on a coral reef ecosystem. Marine Ecology Progress Series 5: 229-241.

Chapman, V.J. 1961. The marine algae of Jamaica. Part Isla Myxophyceae and Chlorophyceae. Bulletin Institute Jaimaica Science Series. 1: 1-159.

Collado-Vides, L. \& J. GonzÁlez-González. 1993. Macroalgas del Sistema Lagunar de Nichupté, Quintana Roo. En: S.L. Salazar-Vallejo \& N. E. González (eds.), Biodiversidad Marina y Costera de México. Comisión Nacional para el Conocimiento y Aprovechamiento de la Biodiversidad y el Centro de Investigaciones de Quintana Roo, México, 752$760 \mathrm{pp}$.

Connell, J. H. 1976. Competitive interaction and the species diversity of coral. In: G.O. Mackie (ed.). Coelenterata ecology and behavior. Plenum Press. New York. pp. 51-58.

Crossland, C.J., B.G. Hatcher., M.J. Atkinson \& S.V. Smith. 1984. Dissolved nutrients of the high latitude coral reef Hourtman Albrolhos Islands, Western Australia. Marine Biology Progress Series 14: 159-163.

Ezer, T, Dv. Thatai, B. KJerfve \& W.D. Heyman. 2005. On the variability of the flow along the Meso-American Barrier Reef system: a numerical model study of the influence of the Caribbean current and eddies. Ocean Dynamics 55: 458-475.

García-Salgado, M., L.T. Camarena, B.G. Gold, M. Vásquez, G. Galland, M.G. Nava, D.G. Alarcón \& M.V. Ceja. 2006. Línea Base del Estado del Sistema Arrecifal Mesoamericano. Proyecto para la Conservación y uso sostenible del Sistema Arrecifal Mesoamericano SAM. Documento Técnico del SAM. $\mathrm{N}^{\circ} 18.167 \mathrm{pp}$.

Garza-Barrientos, M.A. 1975. Primeras consideraciones referentes sobre la flora marina del sureste de la República Mexicana. Memorias del 11 Simposio Latinoamericano sobre Oceanografía Biológica. Universidad de Oriente. Cumaná, Venezuela. 7-25 pp.

HATCHER, B.G. 1985. Ecological research at the Houtman's Abrolhos: High latitude reefs of western Australia. Proceeding of the Fifth International Coral Reef Congress 6: 291-297.

Huerta-Múzquiz, L. 1958. Contribución al conocimiento de las algas de los bajos de Campeche, Cozumel e Isla Mujeres.
Anales de la Escuela Nacional de Ciencias Biológicas, México 9(1-4): 115-123.

Huerta Múzquiz, L. 1986. Algas marinas poco comunes de la flora mexicana IV Crovania attenuata (Bonnemaison) J. Agardh (Rhodophycophyta Fam. Ceramiaceae). Phytologia 60(6): 443-445.

Huerta-Múzquiz, L., A.C. Mendoza- GonzÁlez \& L.E. Mateo-Cid. 1987. Avance sobre un estudio de las algas marinas de la península de Yucatán. Phytologia 62(1): 23-53.

Hughes, T.P. 1994. Catastrophes, phase shifts, and large-scale degradation of a Caribbean coral reef. Science 265: 15471551.

Hughes, T., A. M. Szmant, R. Steneck, R. Carpenter \& S. Miller. 1999. Algal blooms on coral reefs: what are the causes? Limnology and Oceanography 44: 1583-1586.

INE. 1996. Programa de Manejo Reserva de la Biosfera de Sian Ka'an. INE/SEMARNAP. Programas de Manejo 3, Áreas Naturales Protegidas, México. 75 pp.

INE. 1997. Programa de Manejo del Parque Nacional Isla Contoy. México. INE/SEMARNAP, México. 123 pp.

INE. 1998a. Programa de Manejo Parque Marino Nacional Costa Occidental de Isla Mujeres, Punta Cancún y Punta Nizuc. INE/SEMARNAP, México. 159 pp.

INE. 1998b. Programa de Manejo Parque Marino Nacional Arrecifes de Cozumel. INE/SEMARNAP, México. 164 pp.

INE. 2000. Programa de Manejo de la Reserva de la Biosfera de Banco Chinchorro. INE/SEMARNAP, México. 200 pp.

Islebe, G.A, N. Torrescano-Valle, M. Valdez-Hernández, M. TuZNovelo \& H. Weissenberger. 2009. Efectos del impacto del huracán Dean en la vegetación del sureste de Quintana Roo, México. Foresta Veracruzana 11: 1-6.

Jordán, E., M. Angot \& R. DE LA ToRre. 1978. Prospección biológica de la Laguna de Nichupté, Cancún, Quintana Roo, México, Anales del Centro de Ciencias del Mar y Limnología. UNAM, 5(1): 179-188.

KeEney, T.S. 1999. Coral Reef macroalgae in northern Sian Ka'an Biosphere Reserve, Quintana Roo, Mexico. M.S. Thesis. Biology Program, Texas A \& M University-Corpus Christi, Corpus Christi, Texas. 58 pp.

LAPOINTE, B.E. 1997. Nutrient thresholds for bottom-up control of macroalgal blooms on coral reefs in Jamaica and southeast Florida. Limnology and Oceanography 42: 1119-1131.

Liddell, W.D., S.L. Ohlhorst \& S.K. Boss. 1988. The significance of Halimeda as a space-occupier and sediment-producer 1-750 m, North Jamaica. Proceeding of the $6^{\text {th }}$ International Coral Reef Symposium. 127-132.

Littler, M.M. \& D.S. LitTleR. 1985. Factors controlling relative dominance of primary producers on biotic reefs. Proceeding of the $5^{\text {th }}$ International Coral Reef Congress 5: 35-39.

Littler, M.N., P.R. TAYLor, D.S. Littler, R.H. Sims \& J.N. Norris, 1987. Dominant macrophyta standing stocks, productivity and community structure on a Belizean Barrier Reef. Atollon Research Bulletin 302: 1-24.

LittLer, D.S., M.M. LitTLer, K.E. Bucher \& J.N. Norris. 1989. Marine plants of the Caribbean. A field guide from Florida to Brazil. Smithsonian Institution Press. Washington D.C. 263 pp.

Littler, M.N., D.S. Littler \& B.E. Lapointe. 1993. Modification of tropical reef community structure due to cultural eutrophication: the Southwest coast of Martinique. Proceeding of the $7^{\text {th }}$ International Coral Reef Symposium 1: 335-343. 
Gayana Bot. 72(1), 2015

Lugo, A.E, C.S. Rogers \& S.W. Nixon. 2000. Hurricanes, coral reefs and rainforests: resistance, ruin and recovery in the Caribbean. Ambio 29: 106-114.

MagurRan, A.E. 1988 Ecological diversity and its measurement. Croom Helm Limited, London. 179 pp.

Marques, C.M. 1998. Probabilidad y estadística para ciencias químico-biológicas. McGraw-Hill, Interamericana de México. 657 pp.

MatA, J.L. 1981. Disturbio y sucesión ecológica en las poblaciones de macroalgas en un arrecife coralino del mar Caribe. VII Simposio Latinoamericano sobre Oceanografía Biológica. Acapulco, México. 499-520.

Mateo-Cid, L.E. \& A.C. Mendoza-GonZÁlez. 1991. Algas marinas bénticas de la Isla Cozumel, Quintana Roo, México. Acta Botánica Mexicana 16: 57-87.

Mendoza-GonzÁlez, A.C. \& L.E. Mateo-Cid. 1992. Algas marinas bentónicas de Isla Mujeres, Quintana Roo, México. Acta Botánica Mexicana 19: 37-62.

NúÑEz-LaRA, E. \& J.E. ArIas-GonZÁlez. 1988. The relationship between fish community structure and environmental variables in the southern Mexican Caribbean. Journal of Fish Biology 53: 209-221.

OrLóCI, L. 1978. Multivariate analysis in vegetation research. Sec. Ed. Dr. W.J. Junk Publ. The Hague, The Netherlands. $187 \mathrm{pp}$.
Pielou, E.C. 1984. The interpretation of ecological data. A premier on classifications and ordination. Wiley Interscience Publ. New York. 263 pp.

Statsoft, Inc. 2010. Statistica 9 for Windows. Tulsa, E.U.

TAYLOR, W.R. 1972. Marine algae of the Smithsonian-Bredin expedition to Yucatan - 1960. Bulletin of Marine Science 22: 34-44.

Torruco, D. 1995. Ecología y Faunística de los corales escleractinios en los arrecifes de coral del sureste de México. Thesis Phd. Universidad de Barcelona, España. 210 pp.

Torruco, D., A. GonzÁlez \& J. Ordaz. 2003. The role of environmental variables in the lagoon coral community structure on the Chinchorro Bank, México. Bulletin of Marine Science 73(1): 23-36.

Torruco, D., M.A. González \& A.D. Torruco. 2013. Las playas de Quintana Roo: Sus riesgos y vulnerabilidad. Periplo Sustentable 14(24): 165-172.

TunnichfFe, V. 1983. Caribbean staghorn coral population prehurricane Allen condition in Discovery Bay, Jamaica. Bulletin Marine Science 3: 132-151.

Wynne, M. 2002. Turbinaria foliosa sp. Nov (Fucales, Phaeophyceae) From the Sultanate of Oman, with a census of currently recognized species in the genus Turbinata. Phycological Research 50: 283-293.

ZAR, J.H. 1999. Biostatistical analysis. Fourth edition. Prentice-Hall, Upper Saddle River, New Jersey, USA. 663 pp.

Recibido: 11.03.14

Aceptado: 19.03.15 